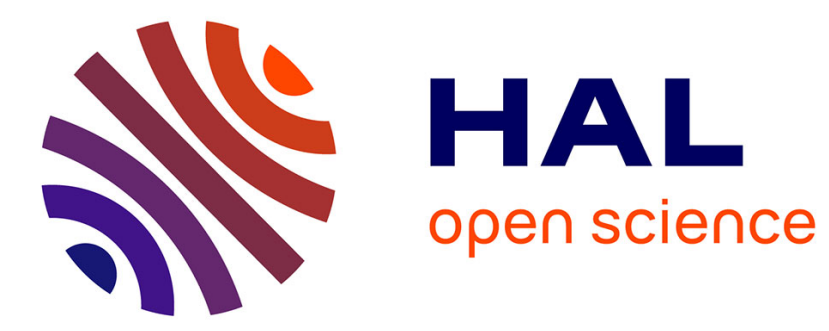

\title{
Cannabis: du produit aux usages. Fumeurs de haschich dans des cités de la banlieue parisienne
}

Sylvain Aquatias

\section{To cite this version:}

Sylvain Aquatias. Cannabis: du produit aux usages. Fumeurs de haschich dans des cités de la banlieue parisienne. Sociétés contemporaines, 1999, 36, pp.53 - 66. 10.3406/socco.1999.1730 . hal-02424166

\section{HAL Id: hal-02424166 \\ https://hal.science/hal-02424166}

Submitted on 26 Dec 2019

HAL is a multi-disciplinary open access archive for the deposit and dissemination of scientific research documents, whether they are published or not. The documents may come from teaching and research institutions in France or abroad, or from public or private research centers.
L'archive ouverte pluridisciplinaire HAL, est destinée au dépôt et à la diffusion de documents scientifiques de niveau recherche, publiés ou non, émanant des établissements d'enseignement et de recherche français ou étrangers, des laboratoires publics ou privés. 


\section{Cannabis : du produit aux usages. Fumeurs de haschich dans} des cités de la banlieue parisienne

Sylvain Aquatias

\section{Citer ce document / Cite this document :}

Aquatias Sylvain. Cannabis : du produit aux usages. Fumeurs de haschich dans des cités de la banlieue parisienne. In: Sociétés contemporaines $\mathrm{N}^{\circ} 36,1999$. pp. 53-66;

doi : https://doi.org/10.3406/socco.1999.1730

https://www.persee.fr/doc/socco_1150-1944_1999_num_36_1_1730

Fichier pdf généré le 01/04/2019 


\title{
Résumé
}

L'observation ethnographique de jeunes fumeurs de cannabis dans des cités de banlieue parisienne montre comment les méthodes de consommation sont liées au contrôle social des familles dans la cité. La dissimulation, mais aussi le contrôle de soi, deviennent des règles informelles dans les groupes de fumeurs de cannabis. Les conduites d'excès ne sont cependant pas absentes et prennent corps dans les longues périodes de désoeuvrement et d'ennui ou dans les occasions à caractère festif. Les conditions sociales dans lesquelles se trouvent ces jeunes gens jouent directement sur les différentes périodes de consommation, qu'elles soient régulées ou excessives. Le travail et les relations sociales entretenues dans la cité influent sur les modifications des consommations dans les parcours. La facilité avec laquelle les fumeurs passent d'un type de consommation à un autre montre que le cannabis est une «drogue molle» dont les usages peuvent être aussi bien doux que durs.

\begin{abstract}
SYLVAIN AQUATIAS

The cannabis and his uses. Hashish smokers in Parisian suburbs

The ethnological observation of young hashish users in the suburban areas around Paris allows seeing how much these uses depend on the social control of the families. The groups of young people have unwritten rules that dictate to not attract attention. They follow these rules by smoking out of view and by keeping self-control when they are stoned. But excessive uses are present when they get too bored and have to kill time or when they have a party. The social situations are a major factor of the different uses of hashish these young people have. The mode they relate with the neighborhood and the fact they're either working or not influence the regulation or the immoderation of their smoking. The shifting from one use to another is easy. The diversity of the different uses according to the social situations reveals how the smoking of hashish could be adapted to various social contexts, from soft uses to hard uses.
\end{abstract}




\title{
CANNABIS : DU PRODUIT AUX USAGES \\ FUMEURS DE HASCHICH \\ DANS DES CITES DE LA BANLIEUE PARISIENNE
}

\begin{abstract}
RÉSUMÉ : L'observation ethnographique de jeunes fumeurs de cannabis dans des cités de banlieue parisienne montre comment les méthodes de consommation sont liées au contrôle social des familles dans la cité. La dissimulation, mais aussi le contrôle de soi, deviennent des règles informelles dans les groupes de fumeurs de cannabis. Les conduites d'excès ne sont cependant pas absentes et prennent corps dans les longues périodes de désœuvrement et d'ennui ou dans les occasions à caractère festif. Les conditions sociales dans lesquelles se trouvent ces jeunes gens jouent directement sur les différentes périodes de consommation, qu'elles soient régulées ou excessives. Le travail et les relations sociales entretenues dans la cité influent sur les modifications des consommations dans les parcours. La facilité avec laquelle les fumeurs passent d'un type de consommation à un autre montre que le cannabis est une "drogue molle » dont les usages peuvent être aussi bien doux que durs.
\end{abstract}

Les critères qui déterminent l'admissibilité d'une drogue dans un pays sont multiples et tiennent autant à la culture et à l'histoire qu'aux dépendances qu'elle est susceptible de provoquer. Ce qui fait craindre les usages de substances psychotropes, c'est bien qu'ils peuvent entraîner des personnes à ne plus respecter les règles de la société, ce d'autant plus que l'usage des drogues, dans les sociétés occidentales modernes, est lié à des représentations de la jeunesse contestataire ou/et marginalisée. Or il est un produit qui pose bien des problèmes aux législateurs puisqu'il est réputé être une «drogue douce» : le cannabis. Le statut ambivalent de cette plante dans l'opinion publique en fait un enjeu considérable pour les tenants de la dépénalisation ou de la légalisation comme pour ceux de la prohibition des drogues. La remise en cause des politiques répressives des drogues, les usages du cannabis en milieu sportif, les prises de position de certaines personnalités l'ont ramené au centre du débat politique. Deux tendances s'opposent : d'un côté, certains décrient le cannabis dont la consommation constituerait un premier pas dans l'engrenage irrémédiable des addictions, de l'autre, l'usage de la marijuana est décrit comme sociable, doux et convivial ${ }^{1}$.

1. On pourrait ainsi opposer les visions respectives de Gabriel Nahas (1976) et de Jean-Pierre Galland (1991) ou même de Martine Xiberras (1989).

Sociétés Contemporaines (1999) $n^{\circ} 36$ (p. 53-66) 
Mais que sait-on réellement des pratiques cannabiques? Dès que l'on travaille dans un champ de pratiques que les normes sociales et les règles juridiques contraignent, les discours et les pratiques diffèrent parfois de manière importante. Comment ne pas s'interroger sur les marges d'erreur des enquêtes quantitatives où la relation de confiance entre l'enquêteur et l'enquêté est minimale ? Le sondage CFES, réalisé pour le Baromètre Santé 1992, a montré, à travers les problèmes de préparation de l'enquête, que les questions portant sur les usages de drogue restent suffisamment sensibles dans la population française pour que les personnes interrogées hésitent à répondre en citant clairement les produits utilisés (Van Solinge, 1995). De même René Padieu note, à propos des enquêtes en milieu scolaire réalisées pour l'INSERM (Choquet et Ledoux, 1989 et 1994, Fréjaville, Davidson et Choquet, 1989), comment celles-ci " ont toutes les vertus souhaitées d'une enquête en population générale : sous réserve d'obtenir la sincérité des sujets, elles donnent une mesure correcte de l'usage de drogue (...) » (Padieu, 1994 ; 30). Mais le caractère illicite des drogues, le cadre institutionnel (en milieu scolaire) de l'enquête peuvent-ils permettre une franchise absolue ? Comment savoir si les usages réels ne sont pas revus à la baisse dans le cadre de l'interaction enquêteur-enquêté qui ne peut échapper à un certain rapport de force ?

Il ne s'agit pas ici de contester l'apport des enquêtes de type quantitatif, seules capables d'apporter les bases d'une évaluation en termes de prévalence. Mais leur efficacité reste limitée quand il s'agit de définir les différents usages du cannabis, les quantités utilisées, la fréquence de consommation, toutes choses nécessaires pour mesurer l'impact réel du cannabis dans la société française. Il faut alors faire appel à des données plus qualitatives pour comprendre les logiques de consommation du haschich. L'approche ethnographique propose ainsi un certain nombre de solutions qui, à défaut de présenter des tendances mesurables, permet de préciser certains aspects des pratiques cannabiques. C'est en adoptant cette méthode que nous avons procédé à une étude des usages du cannabis dans des cités de banlieue de la SeineSaint-Denis d'octobre 1994 à février 1997.

Le fait même de choisir d'étudier les usages du cannabis en banlieue, et notamment dans des cités d'habitat social que l'on peut qualifier de défavorisées, va à l'encontre du profil-type du consommateur de cannabis tel qu'il nous est souvent décrit $^{2}$. Pour avoir déjà travaillé dans des cités de banlieue, nous savions que le haschich y était fort répandu. Et de même savions-nous que l'accès aux informations, dans un milieu où la méfiance est grande vis-à-vis des « gens de l'extérieur », allait être difficile. Nous avons donc choisi de procéder par ce qu'il est coutume d'appeler « observation participante», en nous mêlant en soirée aux groupes de fumeurs dans les cités.

2. Si on en croit un sondage SOFRES de Mai 1992, le profil type du consommateur de cannabis est un homme de moins de 25 ans appartenant aux catégories aisées de la population et habitant la région parisienne ou le Sud-est de la France. On peut bien sûr émettre des réserves sur ce type de travaux, mais cette vision des choses se retrouve dans l'étude plus récente de l'IREP qui donne à voir une proportion importante de fumeurs de cannabis vivant de manière autonome $(73 \%$ sur un échantillon total de 1087 individus), disposant d'une insertion professionnelle (66\%) ayant un niveau d'études correct ou étant encore étudiants. Le choix d'une méthode dite «boule de neige » explique certainement ce biais (Ingold Toussirt, 1998). 


\section{NOTE METHODOLOGIQUE}

L'approche ethnographique, en ce qui concerne cet objet de recherche, pose un certain nombre de problèmes méthodologiques. Afin de nous insérer dans le milieu local, nous avons acheté du cannabis et, nous saisissant de ce prétexte, nous nous sommes petit à petit installés dans les groupes et avons partagé leurs discussions au rythme de deux soirs par semaine environ. En nous présentant d'abord comme des acheteurs afin d'accéder aux groupes, nous étions bien obligés de consommer, aussi peu que ce soit. Afin d'échapper aux effets du cannabis nous avons procédé de différentes manières : en mettant aussi peu de cannabis que possible dans les cigarettes qui nous étaient destinées, en les laissant se consumer, etc. Ces techniques sont rendues possibles par le fait que, la plupart du temps, les cigarettes de haschich sont personnelles et ne passent pas entre les mains d'autres fumeurs. Ainsi les effets du produit sont restés marginaux et n'ont pas été une gêne pour l'observation. Le fait de partager des activités communes avec les personnes, sur place, permet assez vite de dépasser les méfiances inhérentes à ces milieux. De fait, nous avons recueilli de nombreuses données sur les consommations. Nous n'avons pas pu enregistrer ou prendre de notes. Les discours rapportés ne sont donc pas des formulations exactes, mais des reconstitutions visant à reproduire au plus près les termes utilisés. Tous les extraits de conversation sont tirés du carnet de bord. Celui-ci était tenu au jour le jour et rapporte l'ensemble des données, qu'elles aient ou non à voir avec les usages de cannabis.

Les données ont été traitées en fonction de thèmes récurrents qui émergeaient nettement de l'observation. A mi-enquête, tous les éléments du carnet de bord ont été codés et rapportés sur une grille recensant tous les discours et les observations. Les différentes hypothèses ont ensuite été testées sur le terrain. La grille était complétée au fur et à mesure des observations et des entretiens.

Trois méthodes différentes d'analyse ont été employées selon que les indicateurs étaient plus ou moins objectivables et mesurables (notre méthode peut être reliée à ces propos de Jean Peneff : "Quelles sont les éléments qui fondent la validité des démonstrations dans les recherches basées sur l'observation directe ? Une des réponses se trouve dans l'ampleur des mesures et des calculs... » Peneff, 1995, pages 119-138.)

- De nombreux échantillons ont été pesés, certains d'entre eux analysés. De même nous avons essayé d'évaluer le nombre des échanges et leur gain sur certains points de vente. Mais aussi nous avons compté, pour chaque soirée, le nombre de personnes présentes sur place, opération d'autant plus difficile que les déplacements sont constants, et évalué, dans les soirées où la consommation était collective, les quantités de haschich consommé en multipliant le nombre de joints par un dosage moyen de cannabis.

- En ce qui concerne le contrôle des jeunes entre eux et les modes de consommation, nous avons d'abord observé leurs récurrences (par exemple, le nombre de remarques et leur insistance lorsque les personnes étaient en état d'ivresse cannabi- 
que), puis nous avons testé nos hypothèses (en faisant nous-mêmes des remarques ou en simulant des comportements d'excès), enfin, nous en avons discuté en fin de terrain, avec ceux que nous connaissions le mieux, afin de confirmer les hypothèses.

- Nous avons aussi accompli un certain nombre d'entretiens et recueilli des données sur le parcours des jeunes afin de comprendre le contexte des conduites d'excès. En rapportant certaines consommations à leur contexte relationnel, économique ou social, nous avons pu dégager des correspondances entre les périodes de désœuvrement et de travail, les difficultés relationnelles dans les familles ou entre jeunes et les types de consommation. Nous avons de même évalué, en recensant les discours indirects sur les personnes et les modes d'adresse à celles-ci, l'état de leurs « réputations » afin de mieux comprendre les arbitrages relationnels dans les cités.

L'étude a porté de manière approfondie sur deux cités mais a concerné une dizaine de cités en tout.

Toutes ces cités, hormis une, regroupent une population relativement pauvre avec des taux de loyers impayés importants, un taux de chômage variant entre 15 et $18 \%$, une proportion élevée de familles nombreuses et monoparentales. La plupart ont connu des réhabilitations et certaines sont intégrées à des contrats de ville. Les familles d'origine maghrébine sont très représentées dans toutes ces cités ${ }^{3}$, sauf une dans laquelle la population est majoritairement originaire d'Afrique noire et une dans laquelle les populations sont très mélangées. Leurs tailles varient beaucoup, la plus petite regroupant six cents personnes, la plus grande presque six mille.

Dans ces cités, la proportion de 15-24 ans représente environ 15\% de la population, et, souvent, le chômage touche ces jeunes de manière importante (jusqu'à $26 \%$ des 15-24 ans). L'âge des fumeurs - et des non-fumeurs - qui se retrouvent en bas des barres varie entre 16-17 ans et 30 ans environ. Cependant, la majorité a entre 1617 et 23-24 ans, c'est-à-dire les âges où ils ne sont plus à l'école et ne travaillent pas encore. Les groupes sont essentiellement composés de garçons. Bien que l'on puisse aussi trouver des filles qui fument avec les garçons, ces cas sont relativement marginaux et, dans les cités où nous avons travaillé, peu de filles sont présentes dans les groupes. Certaines consomment aussi du cannabis, mais davantage dans un cadre privé.

De même, si les groupes réunissent dans la journée essentiellement des jeunes n'allant plus à l'école et ne travaillant pas, en soirée on trouve aussi des jeunes - et de moins jeunes - travailleurs. A quelques exceptions près, les jeunes travailleurs rencontrés occupent des emplois précaires dans la maintenance, le transport, le nettoyage, le bâtiment et le magasinage.

3. La sur-représentation des jeunes d'origine algérienne dans ce texte est du à la fois au choix des extraits, mais aussi à la forte présence de ces jeunes dans les cités étudiées. Cependant, nous avons aussi rencontré des jeunes d'autres origines, y compris des français. Enfin on trouve dans les groupes de jeunes et ce quelle que soit l'origine aussi bien des fumeurs de cannabis que des nonfumeurs. La consommation de cannabis, telle que nous l'avons observée dans les cités, n'est absolument pas liée à un aspect ethnique ou culturel. 


\section{DU CONTROLE SOCIAL LOCAL AUX REGLES DE LA CONSOMMATION DANS LES GROUPES}

On ne peut comprendre les différents usages du cannabis si on ne les resitue pas dans le contexte global dans lequel ils prennent corps. Si l'exemple des jeunes de banlieue nous renseigne sur les usages du cannabis, les usages du cannabis en banlieue nous renseignent sur la vie des jeunes des cités. Aussi, avant de pouvoir décrire les pratiques, il est nécessaire de décrire brièvement les contraintes qui s'exercent dans les cités de banlieue sur les habitants et ensuite, plus précisément, sur les fumeurs de cannabis.

Les jugements trop souvent négatifs que les institutions, les médias, les autres citoyens portent sur les cités de banlieue les regroupent dans un univers indifférencié : habiter en banlieue, c'est faire partie d'une catégorie stigmatisée qui nivelle les trajectoires sociales particulières. Pour combattre cette indistinction qui nie leurs identités propres, les habitants se saisissent des éléments même de la stigmatisation pour créer des hiérarchies locales, fluctuantes et indécises, basées essentiellement sur les capacités éducatives des parents. Les analyses de Monique Selim et de Gérard Althabe ont bien montré comment les relations sociales des cités procédaient d'un « (...) échange généralisé d'accusations, mettant en jeu de manière permanente une situation de procès dont la matière est trouvée dans les relations internes à la cellule familiale » (Althabe, 1985 ; Selim, 1979). Les familles se jugent entre elles, à partir des relations intra-familiales et des comportements de leurs enfants, reproduisant ainsi à l'échelon local le procès que leur fait la société française à travers les discours des médias, les contacts avec les représentants de l'institution scolaire et les travailleurs sociaux. Les déviances juvéniles ne sont pas les seuls éléments qui permettent de bâtir les différenciations entre familles dans la cité, mais elles sont la matière la plus évidente, la plus visible des dysfonctionnements familiaux. Elles mettent en cause la surface «publique » de la famille : les attitudes des enfants peuvent faire condamner les parents dont les carences éducatives sont ainsi désignées. Dans la cité il est nécessaire de prouver aux autres familles que l'on «tient » ses enfants : «Parce que dans le champ résidentiel des HLM, enfants et adolescents peuvent occasionner un certain désordre, du simple fait de leur visibilité, la responsabilité des familles, leur sens éducatif est au cour du procès » (Calogirou, 1989 ; 40). Les parents, s'ils ne sont pas toujours physiquement présents en bas des immeubles, surveillent depuis les fenêtres les actes des jeunes.

Les enfants connaissent et acceptent les enjeux que représentent les réputations dans les cités. Non pas qu'ils modifient leurs actes pour qu'ils correspondent aux désirs parentaux : le vécu des jeunes est par trop différent de celui des parents pour qu'il y ait possibilité de concilier les deux, hormis en quelques occasions. Mais ils tentent de masquer leurs déviances les plus gênantes, celles qui pourraient disqualifier la famille dans l'espace local.

Cet agencement particulier des relations sociales, à la fois dans la famille et dans la cité, joue sur les modes de consommation du cannabis. Ainsi les jeunes qui habi- 
tent encore chez leurs parents ${ }^{4}$ ne fument que très rarement au domicile de ceux-ci : le risque de se faire prendre sur le fait est trop important. Il ne leur reste alors que les espaces communs des cités pour se retrouver. Encore faut-il qu'ils puissent échapper aux regards des membres de leurs familles ou de toute personne qui pourrait venir les informer sur leur attitude. Aussi les halls et les entrées d'immeubles où ils se retrouvent sont-ils en général éloignés de ceux où résident leurs parents. Le fait d'accaparer des caves et certains locaux fait partie de cette logique de dissimulation.

Quand ils fument sur les espaces extérieurs de la cité, les jeunes, sachant qu'ils risquent d'être vus, préparent leurs joints le plus vite possible. En été, quand il fait beau et que les gars ont envie de rester dehors, il est fréquent d'en voir un se diriger sous un arbre ou rentrer dans un hall, le temps de rouler son joint, avant de revenir vers ses copains pour le fumer. Il n'est pas question ici de confectionner le cône traditionnel assemblé avec plusieurs feuilles de papier à rouler : on vide simplement le contenu d'une cigarette dans une seule feuille de papier sur laquelle on émiette rapidement le cannabis, le joint ainsi fait ressemblant le plus possible à une cigarette.

S'ils veulent éviter d'être repérés par leurs parents depuis leurs fenêtres, les jeunes doivent aussi éviter les comportements qui feraient trop remarquer leur état : il est de bon ton de garder la face en public et de ne jamais avoir un comportement qui indique que l'on est " défoncé ». Il faut garder le contrôle de soi, à la fois pour éviter d'être désigné comme un fumeur de haschich (par rapport à la famille et à certains habitants) et comme un mauvais fumeur (dans le groupe de jeunes). Les gars se surveillent mutuellement : que l'un d'entre eux prépare trop ouvertement son joint, que ses yeux soient trop rouges ou que son comportement montre par trop son état, et les autres lui feront remarquer qu'il dépasse les limites. La surveillance qu'ils exercent les uns sur les autres répercute le contrôle social des familles dans les groupes. Des règles, informelles certes, mais s'appliquant avec une certaine rigueur, balisent les comportements des fumeurs de cannabis.

Pourtant, s'ils fument en groupe, leurs usages sont essentiellement - mais pas uniquement - individualistes. Les joints circulent peu. Si un gars désire partager avec un copain, il lui tendra plus volontiers un bout de résine détaché d'une barrette pour qu'il se roule son propre joint. Ce comportement pourrait être expliqué par la volonté de se dissimuler vis-à-vis des personnes passant dans les halls ou pouvant les apercevoir des fenêtres : si un jeune qui fume sa cigarette n'est pas forcément un consommateur de cannabis, par contre, une cigarette qui tourne entre plusieurs jeunes évoque irrésistiblement l'idée de la drogue.

Mais on voit bien dans la précipitation avec laquelle ceux qui reviennent du travail ou du lycée roulent leur première cigarette de haschich, dans les réticences de ceux à qui il est réclamé une bouffée de fumée euphorisante, dans la manière dont certains s'isolent pour éviter les demandes de ceux qui sont dépourvus de cannabis, qu'il s'agit au moins autant de préserver sa propre consommation. Le partage ne se fait que plus tard dans la soirée quand les fumeurs sont plus détendus et ont leur comptant de cannabis. Même alors, la circulation des joints entre les fumeurs

4. Ils représentent la majorité des personnes rencontrées. Du fait des restrictions d'accès à l'indépendance financière et d'un parc immobilier souvent saturé, on trouve fréquemment des personnes de trente ans et plus habitant encore chez leurs parents. 
n'englobe pas la totalité des personnes présentes et montre ainsi l'état des relations qu'elles entretiennent. Les relations préférentielles se déclinent depuis le premier fumeur, celui qui a roulé, jusqu'au dernier, celui à qui on passe un joint presque consumé. La personne à qui le premier fumeur passe son joint est quelqu'un avec qui celui-ci s'entend davantage qu'avec les autres. Le premier fumeur garde un droit de regard, une fois qu'il a passé le joint, sur les personnes qui vont rentrer dans le tour ${ }^{5}$. Souvent il désigne au second fumeur d'un mouvement de tête la personne à qui il devra ensuite passer la cigarette. C'est le premier fumeur qui détermine à qui la cigarette va être passée ou pas et il peut restreindre l'étendue du tour. Il nous est souvent arrivé d'entendre le premier fumeur protester alors qu'un de ceux à qui il avait passé le joint le tendait à un autre et réclamer qu'on le lui redonne. Dans un seul et même groupe de jeunes, plusieurs joints peuvent tourner qui suivent un circuit précis et il arrive même que certains soient exclus de ces tours.

D'une part fumer du cannabis suit des règles bien précises qui impliquent un certain contrôle de soi, et d'autre part le cannabis est un produit important, qui ne se partage pas de n'importe quelle manière et dont l'usage est personnel avant d'être collectif. Pourtant, si les régulations de la consommation apparaissent nettement dans les groupes, elles ne peuvent dissimuler les consommations parfois intensives des jeunes.

\section{CONDUITES D’EXCES : LE FESTIF ET L'ENNUI}

Parfois, un groupe se retrouve dans un hall ou un local. Tout le monde a de quoi fumer et tout le monde fume. Chacun y va de sa meilleure histoire. On rit beaucoup, à propos de tout et n'importe quoi. Parfois, l'un d'eux a à peine passé le joint à son voisin qu'un autre arrive. On voit parfois un gars avec deux joints, un dans chaque main, hésitant, ne sachant à qui le tendre. Dans la soirée, peuvent ainsi défiler vingtcinq à trente joints pour six à sept personnes. Les cigarettes elles-mêmes marquent le caractère festif de la soirée. On voit apparaître des joints roulés avec deux ou trois feuilles munis de filtres en carton. Ce n'est pas le petit joint que l'on roule en bas de l'immeuble en regardant autour de soi, avec une seule feuille, vite fait. Celui-là, on va « se poser» pour le faire, sur une table ou une chaise. On peut comparer la soirée à une bringue entre amis, à une joyeuse soûlographie. On s'attarde, personne n'a envie de partir. Et avant de s'en aller, puisqu'il faut bien rentrer, on va faire un dernier petit joint, comme en d'autres lieux on boirait le coup de l'étrier, " pour la route ».

La consommation varie non seulement selon le nombre de personnes, mais aussi selon la qualité des produits ${ }^{6}$. Dans certaines soirées, cela peut représenter une consommation d'environ deux grammes par personne, soit l'équivalent d'une barrette courante de cannabis, c'est-à-dire ce qu'un consommateur moyen (trois joints par jour) va consommer en trois à quatre jours. Cette évaluation ne nous permet pas de juger réellement de l'excès, les situations pouvant être fort différentes d'une soi-

5. J'utilise ici l'expression « tour » pour désigner la circulation complète d'un joint collectif, du premier fumeur - celui qui, en général, l'a roulé - au dernier - celui qui l'a écrasé.

6. Les échantillons recueillis sur notre terrain d'enquête montrent une gamme de produits allant de $3,52 \%$ à 18,63\% de THC. Analyses effectuées par le professeur Gilbert Fournier du laboratoire de pharmacognosie de l'université de Chatenay-Malabry. 
rée à l'autre, selon le nombre de personnes, la quantité et la qualité du produit; elle nous donne simplement à voir le dépassement possible par rapport à une consommation moyenne.

Une autre figure de l'excès apparaît, lors des longues journées de galère où rien ne se passe, où l'on «zone » et où l'on attend. Ceux qui se retrouvent en bas des immeubles au début de l'après-midi ne travaillent pas et ne vont plus à l'école. L'inactivité les ronge et la monotonie des jours qui défilent semble ne jamais devoir être rompue. Ils dorment le plus longtemps possible, ce qui est du aussi au fait qu'ils veillent tard, profitant le plus possible de la soirée, seul moment où les va-et-vient entre les cités et la présence des autres jeunes de la cité meublent l'ennui. A force de se retrouver ensemble, jour après jour, ils n'ont plus grand-chose à se dire. Pour tromper l'ennui, pour introduire un peu d'euphorie dans la grisaille du quotidien, ils fument comme pour «allumer le monde» (Nahoum-Grappe, 1991; 91). Certains d'entre eux ont déjà fumé au réveil. Les joints succèdent aux joints. Une voiture garée à côté du hall ou du banc où s'est réuni le groupe diffuse du rap américain. Le partage est plus présent : ils se trouvent dans une situation de forte dépendance les uns par rapport aux autres, à la fois parce qu'ils ont peu de sociabilités externes à la cité, mais aussi parce que les réseaux de proximité sont les seuls à même de fournir des ressources, légales ou illégales, pour gagner de l'argent. Leur consommation est en partie régulée - on essaie de préserver le contrôle de soi - mais concerne parfois des quantités importantes quand un joint succède à l'autre et que, finalement, ils auront passé leur journée à fumer. Seul le cannabis arrive à ramener un peu de joie dans des discussions qui s'épuisent au fur et à mesure qu'elles se répètent. Il s'agit bien d'une situation collective d'excès : le besoin de fumer, l'enchaînement des joints montrent comment le contrôle de soi s'amenuise dans l'envie collective ${ }^{7}$. S'il arrive qu'un gars de la cité, plus âgé, passe, il n'hésitera pas à faire remarquer aux fumeurs que leur état est excessif.

Il est quasi impossible d'évaluer une consommation moyenne dans ce type de cas, ne serait-ce que parce que la consommation est davantage collective. Certains n'auront fumé que quelques joints, mais d'autres auront parfois utilisé une barrette entière.

Dans les deux cas, les participants admettent qu'il est acceptable de fumer beaucoup et de laisser le contrôle de soi diminuer. C'est parce qu'il s'agit d'usages collectifs qu'on peut dépasser les préventions habituelles. Il est plus facile de ne pas tenir compte d'un commun accord des règles fixées que de les transgresser seul. D'un autre côté, on doit noter que ces scènes de l'excès se passent le plus souvent dans des endroits fermés, caves ou locaux, ou dans des places éloignées de la visibilité des familles.

Bien des usages collectifs se trouvent situés entre ces deux figures. Il est parfois ardu de séparer la simple discussion animée de la soirée à caractère festif, de même qu'il est parfois difficile de séparer l'expérience individuelle de l'expérience collec-

7. II importe de préciser que les observations qui ont permis de décrire ces deux modèles ont été faites avec des groupes d'âge assez différents, sur des cités différentes. Dans le premier cas, il s'agissait de gens ayant entre 22 et 34 ans. Dans le second, de jeunes ayant entre 17 et 25 ans. On peut rapprocher ces faits de la période d'indétermination sociale et de la moindre autonomie, en termes financiers et relationnels, des plus jeunes. 
tive : dans un même groupe, tous ne vivront pas de la même façon un même moment de fumée partagée.

\section{LES CONDITIONS SOCIALES DES CONSOMMATIONS INTENSIVES}

Pourtant, il semble évident que ces consommations conséquentes et quotidiennes sont liées à des conditions sociales. En premier lieu intervient l'absence de statut et d'argent que subissent les jeunes qui, sortis du système scolaire et laissés à l'écart du monde du travail, se retrouvent immobilisés dans leurs cités. Une économie minimale se crée bien qui, entre les petits boulots (stages et missions d'intérim, petits chantiers « au noir ») et les activités illicites, permet d'accéder tant bien que mal aux biens valorisés et valorisants de la société de consommation. Mais l'ennui et la perte des perspectives d'avenir pèsent lourdement sur ces jeunes vivant une indétermination sociale ${ }^{8}$ qui, habituellement transitoire, semble devoir se prolonger à l'infini. Le cannabis devient un recours, un «médicament » contre le désœuvrement et la lassitude.

Cependant, le manque d'endroits où les jeunes puissent disposer d'une relative intimité, le jeu des rumeurs et des réputations dans les cités et la nécessité de recourir à des activités déviantes (consommation de produits illicites, mais aussi activités liées à l'économie souterraine) ont conduit à l'émergence de règles de comportement. L'excès en public ne peut que rentrer dans des cadres sociaux collectivement acceptés, qu'il soit individuel ou collectif. On pourra, par exemple, tolérer que quelqu'un ait une consommation excessive de manière ponctuelle, parce qu'on sait qu'il a des problèmes en ce moment, qu'un évènement ou un autre est arrivé qui l'autorise à se « laisser aller» un peu. Mais si un jeune est sans cesse dans l'excès, il sera assez vite mis à l'écart.

Si les conditions sociales dans lesquelles vivent les jeunes de banlieue accentuent les probabilités d'utilisation intensive, les cas d'excès ponctuels ou prolongés prennent corps dans les histoires particulières des personnes. On verra aussi bien sombrer, pour un soir, dans l'abus de cannabis, un jeune qui a failli se faire prendre par la police après un vol de voiture manqué ; pendant quelques mois celui que sa petite amie a laissé tomber; ou de manière prolongée celui dont la profession est trop stressante. Les logiques de l'excès lient les parcours individuels et les situations sociales. Deux variables apparaissent nettement dans les modifications des consommations : les relations sociales et l'accès au monde du travail.

Les jeunes ont beaucoup de relations sociales avec d'autres jeunes de cités et peu avec des personnes d'autres milieux sociaux; aussi les enjeux des réputations dans les cités sont-ils particulièrement importants. Un garçon qui est disqualifié vis-à-vis des groupes de voisinage ne dispose pas des ressources suffisantes pour faire appel à des relations externes. Dès que les liens qu'une personne entretient avec le groupe commencent à se fragiliser ou à devenir conflictuels, cela a des conséquences sur les quantités fumées, qu'il s'agisse d'augmentation ou de baisse. Ainsi Youssef expli-

8. Cette indétermination sociale est aussi une surdétermination. Surdétermination matérielle d'abord, puisque les équipements collectifs et les services étatiques sont souvent moins présents dans les périphéries urbaines, mais surdétermination symbolique aussi, les stéréotypes des jeunes habitant les cités jouant directement sur leur moindre accès à l'emploi. 
que pourquoi il a réduit sa consommation : «Je voyais bien que ça allait pas et puis, je sais pas, ça te rend bizarre aussi, tu parles mal, avec tout le monde, tu deviens agressif, même avec tes parents, t'en as plus rien à foutre, tu leur réponds et tout.... Non, ça allait plus, alors, dans ma tête, je me suis dit qu'il fallait mieux que je fasse gaffe, alors j'ai arrêté... » (28/Célibataire/travail.)

D'une barrette par jour, il est passé à deux ou trois joints qu'il fume uniquement en soirée. Mais l'inverse peut aussi se produire quand un jeune se retrouve disqualifié dans les groupes de voisinage. Les logiques de l'excès peuvent alors inciter à multiplier les produits et des poly-toxicomanies apparaissent.

La cité où habite Mustapha est petite, trois bâtiments se faisant face, dessinant un carré complété par la rue. Au centre de l'espace ainsi circonscrit, un bac à sable et des jeux pour les petits, quelques bancs... Dès qu'on arrive par la rue, on est sous les yeux des jeunes dans l'ouverture des trois blocs. Ils sont là, ramassés en deux groupes devant les entrées du bâtiment de droite. Nous saluons et demandons Mustapha. Les gars nous le désignent en haussant les épaules, d'un air qui en dit long. Au centre, sur un banc proche du bac à sable, Mustapha et un autre gars sont assis sur le dossier du banc, les pieds sur le siège. Mustapha est explosé, ses yeux cernés ne sont plus que deux fentes qui disparaissent sous le petit bonnet tombant sur son front. Il nous tend une bouteille...

"C'est quoi? " demande Tayeb.

"Du rhum et de l'acide... »

"Non merci, je bois pas d'alcool», dit Tayeb. (Extrait de carnet de bord, 15/05/95, Mustapha : 20/Célibataire/sans travail.)

Que Mustapha s'expose ainsi en plein centre de la cité, " défoncé », une bouteille à la main, montre bien que sa réputation est déjà trop dégradée pour qu'il puisse espérer y changer quelque chose. Qu'il fume ou qu'il boive, il est toujours dans l'excès. Il se moque bien d'être vu ou non. Le fait qu'il soit seul - avec un autre gars - alors que tous les autres sont près des entrées marque sa disqualification sociale.

Le travail aussi agit sur l'intensité des consommations, d'abord parce trouver une place, c'est avant tout accéder à un statut social reconnu et valorisant et donc changer sa position sociale dans la hiérarchie locale, mais aussi parce qu'il implique une structuration différente des temporalités. L'économie souterraine, recours fréquent pour disposer de quelques revenus, épuise vite son pouvoir de séduction.

«Les jeunes dans la banlieue, ils businessent et tout, c'est le bon côté, mais ça dure pas, comment t'es bien quand t'as pris des trois ou quatre piges (de prison)... On croit que c'est que les cabriolets, la Thaïlande... C'est l'autre côté de la plaque... Le business de shit (cannabis), t'as les thunes comme ça, mais c'est toujours les mêmes histoires... C'est pas comme le mec qu'à un travail. Le mec qui a un travail, il se lève pour aller bosser, il y va aussi pour l'argent, mais c'est pas pareil, parce qu'il a quelque chose à faire, tandis que toi, du matin au soir, t'es là à te galérer sur la cité, et à la finale, tu vends toute la journée, à la finale tu te lèves que pour l'argent, alors que celui qui se lève pour travailler, il a quelque chose à faire au moins. Tu vois ce que je veux dire? Hé ben c'est ça, le jeune qui fait son business, il se lève pour l'argent, ça lui plaît ou ça lui plaît pas, il faut bien qu'il fasse 
quelque chose. On se lève tous pour le liquide. Mais en vrai, t'as même pas envie de te lever, parce que tu sais que tout ce que tu vas faire, c'est galérer sur la cité et attendre le client... », dit Malik.

Salif complète : «On fait la pute, c'est le même système, la pute, elle attend son client... »

(Malik, 27/concubinage/1 enfant/sans travail, et Salif, 24/célibataire/sans travail. Respectivement vendeur et ex-vendeur de cannabis.)

Ceux qui vont arriver à accéder au travail légal vont alors découvrir la nécessité de contrôler leurs consommations, mais aussi redécouvrir le plaisir du haschich à travers un changement de rythme.

"Tu vois, ce qui est bien, depuis que je travaille, c'est que les pétards, je les calcule, avant, je fumais, c'était comme ça, j'avais envie, je fumais, maintenant, je travaille, alors tous on fait gaffe, on se dit, il faut chafrav (travailler), alors on attend, et puis quand tu sais que tu vas faire une petite pause, que tu peux t'en allumer un, alors, là, je te garantis, c'est le bonheur, il te fait de l'effet, je me dis que je vais être heureux. »

Comme ça me fait rire, il se retourne vers moi : "Sérieux, Sylvain, c'est trop bon, tu peux pas savoir, tu vois, ce week-end, j'ai fumé, les pétards, je les fume et c'est tout. Là, tu vois, (il me montre la table) il y en a, hé bien, c'est même pas ça, je suis bien, là. " (21/célibataire/travail précaire.)

On voit ainsi des jeunes gens qui ne fument plus qu'en soirée, ou qui, au contraire, fument avant d'aller au travail un joint dosé avec soin de manière à ce que les effets ne soient pas incapacitants.

Mais les régulations n'interviennent réellement que si le travail est suffisamment mobilisateur, c'est-à-dire s'il permet d'obtenir une certaine stabilité et une valorisation sociale minimale. Ceux qui se retrouvent cantonnés à des emplois précaires et peu intéressants limitent bien leur consommation aux soirées, mais ces consommations sont souvent alors conséquentes ${ }^{\text {? }}$.

José, par exemple, dit consommer pour environ mille cinq cents francs par mois de cannabis. Il a tout fait : maçon, électricien, «technicien de surface », cariste : de petits contrats qui ne durent jamais bien longtemps. Il a vingt-neuf ans, vit en couple, a un enfant. Il lui faut assurer le minimum, un minimum auquel le marché de l'emploi ne lui permet pas toujours d'accéder. Aussi, il complète par des petits travaux au noir, vend parfois sa propre production de marijuana. Il est nécessaire qu'il reste intégré au réseau des jeunes de la cité pour bénéficier de débouchés et de « tuyaux » et passe donc beaucoup de temps en soirée à discuter dans les halls.

9. De plus, dans la plupart des cas, ces fumeurs, plus mobiles, souvent un peu plus âgés, ont accès à des réseaux plus diversifiés et sélectionnent des produits plus forts. D'après les analyses que nous avons pu faire, les cannabis utilisés par ces fumeurs contiennent en moyenne $10 \%$ de principe actif alors que les cannabis trouvés couramment sur les cités n'en ont que $6 \%$. 


\section{LE CANNABIS : UNE DROGUE MOLLE ${ }^{10}$}

La vision d'un lien fort entre la consommation de cannabis et les activités adolescentes doit donc être pondérée. Si on ne peut nier, comme l'écrivent Michel Kokoreff et Patrick Mignon, que la consommation de cannabis " (...) fait partie intégrante d'une sociabilité adolescente dont elle est un opérateur important; elle s'intègre pleinement dans une culture de groupe " (Kokoreff, Mignon, $1994 ; 119$ ) le fait qu'elle soit alors liée à un stade de la vie, à un âge, est moins évident. " $A u$ fond on pourrait se demander si arrêter le shit, ce n'est pas marquer un seuil, symboliser son entrée dans la vie adulte en enterrant sa vie d'adolescent et ses déviances les plus apparentes " s'interrogent les auteurs (Kokoreff, Mignon, 1994 ; 138). L'arrêt du cannabis dans les cités, il est vrai, tient souvent de la manipulation symbolique (vis-à-vis des autres jeunes, de la famille, de la cité). Pourtant, non seulement, la plupart du temps, il ne s'agit pas d'arrêt mais plutôt de régulation, mais encore les consommations intensives elles-mêmes peuvent se prolonger. Il ne s'agit pas tant d'une question d'âge que d'accès à une situation sociale stable et satisfaisante.

A ce titre, les effets sociaux des consommations intensives de cannabis ne sont pas négligeables. La mobilité déjà réduite des jeunes dans les cités est encore accentuée par l'apathie que provoquent les excès cannabiques et ceux-là même qui, n'arrivant pas à se décider à accomplir une démarche administrative, fument un joint pour se donner du courage, parfois ne bougent pas de la cité. S'ils arrivent jusqu'aux guichets, les effets désinhibiteurs du cannabis ne joueront pas toujours en leur faveur. Le cannabis n'est pas seul responsable de ces situations et il y aurait beaucoup à dire sur les rapports qu'entretiennent les jeunes et les institutions. Mais les usages excessifs accentuent les problèmes de mobilité et d'insertion. De même, le développement du marché du cannabis dans certaines cités provoque des tensions importantes entre les habitants, les jeunes et la police. La paranoïa guette celui qui, toujours pris dans les brumes du haschich, ne sait plus gérer ses relations sociales.

Pourtant, davantage que du produit, c'est bien de ses usages qu'il est question. Des phases de consommation intensive et des phases de consommation régulée, des phases d'arrêt et des phases d'excès vont s'articuler, constituant des parcours et des relations au produit fort différents. C'est aussi que le cannabis est une drogue molle qui permet de nombreux usages, qui est assez facilement manipulable.

La fréquence des usages intensifs dans les cités où nous avons travaillé est liée à des conditions sociales ${ }^{11}$ : stigmatisation et indétermination, ennui et absence de valorisation.

Peut-on alors penser que les effets recherchés à travers le cannabis sont de l'ordre de ceux recherchés dans la consommation des médicaments psychotropes

10. Je reprends ici le terme employé par Numa Murard dans la postface qu'il a écrite pour le rapport d'enquête.

11. Pourtant, les usages intensifs ne sont pas l'apanage des jeunes de cités. Dans l'enquête de l'IREP déjà citée, $56,12 \%$ des personnes interrogées fument quotidiennement, en moyenne 4,35 joints par jour, mais peuvent aller jusqu'à plus de 20 joints. 50,6\% d'entre elles admettent pouvoir consommer seules, ce qui met à mal la notion de convivialité qu'on associe souvent aux usages récréatifs des classes moyennes (Ingold, Toussirt, 1998). 
utilisés dans d'autres couches de la population française ? Alain Ehrenberg explique cette consommation croissante par la montée du processus d'individualisation : il augmente la tension qui s'exerce sur les personnes et il rend les difficultés quotidiennes sans cesse plus insupportables : "ces difficultés ne peuvent qu'augmenter dans une société de responsabilité de soi, où l'échec scolaire, professionnel ou social est de plus en plus imputé à l'individu lui-même et conduit à des frustrations de masse que ne connaissent pas les sociétés de destin » (Ehrenberg, 1995 ; 150).

Dans les cités, comme partout dans la société française, les mythes du succès social et de la réussite personnelle ont cours. Que l'usage du cannabis se généralise n'étonne alors pas. Cette généralisation marque aussi bien, pour les jeunes, la reconnaissance du principe du mérite personnel que celle des conditions particulières faites aux habitants des banlieues. Les contraintes à l'œuvre sont encore plus fortes, puisque les chances de s'en sortir sont plus faibles.

En ce sens, le cannabis se présente comme une alternative, un pis-aller au stress quotidien. La banalisation et l'extension de sa consommation est un symptôme du mal-être dans les cités de banlieue. Mais, évidemment, comme les médicaments psychotropes, le cannabis peut aussi bien être utilisé comme un médicament que comme une drogue. L'opposition entre drogues dures et drogues douces s'avère inopérante pour classer le cannabis. Comme l'alcool, celui-ci permet de nombreuses utilisations. Et comme l'alcool, certaines de ces utilisations peuvent être dangereuses. Il ne s'agit pas de diaboliser le cannabis mais plus simplement d'affirmer que, comme tout produit psycho-actif, certains de ses usages peuvent poser problème.

Sylvain AQUATIAS

GDR G 1106, «Psychotropes, politique et société » 14, rue Corvisart, 75013 PARIS aqtias@club-internet.fr 


\section{REFERENCES BIBLIOGRAPHIQUES}

Althabe G. 1985. Urbanisation et enjeux quotidiens. Terrains ethnologiques dans la France actuelle. Paris, Anthropos.

Aquatias S., Khedim H., Murard N., Guenfoud K., avec la collaboration de G. FOURNIER. 1997. L'usage dur des drogues douces, recherche sur la consommation de cannabis dans la banlieue parisienne. Paris, Iresco/Grass, Mildt-Mesr.

BoekHOUT VAN SOLINGE T. 1995. Le cannabis en France. Cedro.

CAlogirou C. 1989. Sauver son honneur, rapports sociaux en milieu urbain défavorisé. Paris, l'Harmattan.

Choquet M., LedouX S. 1994. Adolescents, enquête nationale. Paris, Inserm.

CHOQuet M., LedouX S.1989. Les drogues illicites parmi les 11-20 ans et attitudes face au Sida, INSERM, ronéoté.

Ehrenberg A. 1995. L'individu incertain. Paris, Calmann-Levy.

Frejaville J.P., Davidson F., Choquet M. 1977. Les jeunes et la drogue. Paris, PUF.

GALLAND J.P.1991. Fumée clandestine, il était une fois le cannabis. Paris, Éditions du Lézard.

INGOLD R., TOUSSIRT M. 1998, Le cannabis en France. Paris, Anthropos.

KoKOREFF M., MignON P. 1994. La production d'un problème social : drogues et conduites d'excès. Dgldt/Mesr.

NAHAS G. 1976. Haschich, cannabis, marijuana. Paris, PUF.

NAHOUM-GRAPPE V. 1991. La culture de l'ivresse, Paris, Quai Voltaire Histoire.

PADIEU R. 1994. L'information statistique sur les drogues et les toxicomanies. La Documentation Française, Paris.

PENEFF J. 1995. Mesure et contrôle des observations dans le travail de terrain, Sociétés Contemporaines, $\mathrm{n}^{\circ} 21$, P. 119-138.

SELIM M. 1979. Rapports sociaux dans une cité HLM de la banlieue Nord de Paris : le clos Saint-Lazare à Stains, Thèse de sociologie, EHESS.

XIBerRas M. 1989. La société intoxiquée. Méridiens-Kliencksieck, Paris. 